\title{
Laser-induced growth of titanium nitride microcolumns on biased titanium targets
}

\author{
E. György, ${ }^{\text {a) }}$ A. Pérez del Pino, P. Serra, and J.L. Morenza ${ }^{\text {b) }}$ \\ Universitat de Barcelona, Departament de Física Aplicada i Òptica, E-08028 Barcelona, Spain
}

(Received 10 February 2004; accepted 20 August 2004)

\begin{abstract}
Titanium targets with a bias voltage ranging from -500 to $+500 \mathrm{~V}$ were submitted to multipulse high repetition rate $\mathrm{Nd}$ :yttrium aluminum garnet (YAG; $\lambda=1.064 \mu \mathrm{m}$, $\tau \sim 300 \mathrm{~ns}, v=30 \mathrm{kHz}$ ) laser irradiations in nitrogen at intensity values below the single-pulse melting threshold. The morphology of the TiN structures formed under the cumulative action of the laser pulses on the surface of the unbiased and biased targets was investigated by profilometry and scanning electron microscopy. Under these irradiation conditions, a specific columnar surface microrelief developed. The height of the microcolumns reached about $10-15 \mu \mathrm{m}$, and their diameter about 1-2 $\mu \mathrm{m}$. The development of TiN microcolumns was enhanced by the applied bias voltage. The enhancement in the negative biased samples was stronger than that in the positive biased ones.
\end{abstract}

\section{INTRODUCTION}

Laser processing of solid surfaces has been extensively investigated during the last decade because of its large variety of applications in recrystallization, doping, smoothing, hardening, or compound formation. ${ }^{1}$ Moreover, specific irradiation conditions permit the creation of different surface morphologies as laser-induced periodic structures, microcones, or microcolumns. We have recently reported for the first time the growth of titanium nitride microcolumns during the cumulative action of high repetition rate laser pulses. ${ }^{2,3}$ In our experiments, we used a pulsed Nd:yttrium aluminum garnet (YAG; $\lambda=1.064 \mu \mathrm{m}$ ) laser for the irradiation of titanium surfaces in high-pressure nitrogen atmosphere.

Surface morphologies, in some aspects similar to those obtained by us, have been recently reported to develop during laser irradiation of various material surfaces. More precisely, microcolumns have been grown on titanium in low vacuum $(1 \mathrm{~Pa}),{ }^{4}$ on tungsten in low-pressure He gas, ${ }^{5}$ as well as on silicon ${ }^{6,7}$ and ceramic composites ${ }^{8}$ in oxygen-containing atmospheres.

Titanium nitride microcolumns grown on titanium can be useful for certain applications. Indeed, in medical implants it is generally accepted that rough surfaces ensure a better osteointegration than smooth ones do. ${ }^{9}$ In

\footnotetext{
${ }^{a}$ On leave from Institute of Atomic Physics, P.O.Box. MG. 36, Bucharest V, 76900, Romania

b) Address all correspondence to this author.

e-mail: jmorenza@ub.edu

DOI: $10.1557 / J M R .2005 .0021$
}

addition, due to its biocompatibility and bioinertness, titanium nitride coatings can act as efficient diffusion barriers against the metal. On the other hand, recent investigations have also been focused on the field electron-emission properties of the titanium nitride microcolumns. ${ }^{10}$

Our purpose in the present work was the investigation of the effect of negative or positive bias voltages applied to the target on the columns growth process. More precisely, we wanted to see the effect of the external electric field generated by the target bias on the species in the ablation plasma and especially on their preferential redeposition on the columns top where the electric field is the most intense. For that, we performed laser irradiations with and without target bias, with all other experimental conditions (laser beam parameters, irradiation geometry, and ambient nitrogen pressure) kept constant. We could not identify similar studies in the literature, concerning laser-surface interactions and laser-generated columnar surface structure formation in the presence of external electric fields.

\section{EXPERIMENTAL}

The irradiations were conducted in a stainless steel vacuum chamber preliminary pumped down to a residual pressure of $2 \times 10^{-4} \mathrm{~Pa}$ and then filled with pure (less than $3 \mathrm{ppm}$ oxygen contamination) nitrogen at a pressure of $3 \times 10^{4} \mathrm{~Pa}$. We used a Baasel LBI $6000 \mathrm{Nd}$ :YAG $(\lambda=1.064 \mu \mathrm{m})$ laser system (Baasel Lasertech, Germany) generating pulses of $300 \mathrm{~nm}$ duration with nearly Gaussian temporal shape. The spatial intensity distribution was characterized by a nearly Gaussian circular 
intense central zone. The laser beam was incident normally to the target surface. Up to 1500 subsequent laser pulses at a repetition rate of $30 \mathrm{kHz}$ were directed onto the same target location.

The targets submitted to multipulse laser irradiation consisted of $10 \times 10 \times 1 \mathrm{~mm}^{3}$, polished, high-purity (99.6\%), hexagonal phase $\alpha-\mathrm{Ti}$ foils. The total laser beam diameter on the target surface was approximately $500 \mu \mathrm{m}$, and that of the central zone was $120 \mu \mathrm{m}$. The total laser pulse energy was fixed at $2.3 \mathrm{~mJ}$, corresponding to about $1.2 \mathrm{~mJ}$ in the central zone of the beam. Thus, the laser intensity in the central zone was about $35 \mathrm{MW} /$ $\mathrm{cm}^{2}$, and in the surrounding more than one order of magnitude lower. These intensity values are below the singlepulse melting threshold of titanium. ${ }^{11}$

A bias voltage ranging from -500 to $+500 \mathrm{~V}$ was applied to the titanium target. During the experiments performed under biased conditions all other irradiation parameters were identical to those under unbiased ones.

The morphology of the laser irradiated surfaces was investigated by profilometry using a Dektak 3030 stylus profilometer (Veeco Instruments, Inc.), and by scanning electron microscopy (SEM) with Jeol JSM 840 (Japan) and Cambridge S120 microscopes (Cambridge Instruments, UK).

\section{RESULTS}

\section{A. Unbiased titanium targets}

The SEM micrographs of the surface structures obtained with 810 and 1500 successive laser pulses are shown in Fig. 1. The micrographs correspond to the center of the laser-irradiated area imprints. The morphology evolution with the increase of the number of laser pulses from a rippled wavelike structure toward a dense columnar one is clearly visible. The diameters of the columns grown with 1500 laser pulses are about $2 \mu \mathrm{m}$, and their lengths reach about $10 \mu \mathrm{m}$.

The surface topography evolution with the increase of the number of laser pulses was followed by profilometry measurements recorded across the diagonal of the laser imprints (Fig. 2). The profiles show an increase in height with the increase of the number of laser pulses. On the other hand, when the number of laser pulses reaches 1500 , a depression in form of a crater is formed in the center of the laser irradiated area. Nevertheless, the bottom of this crater remains above the non-irradiated target surface. The magnifications in Figs. 1 and 2 are different. Thus, Fig. 1 shows only a part from the center of the whole, about $650-\mu \mathrm{m}$ diameter imprint. Moreover, the structures visible in the surface profiles are higher than the columns in the SEM image (Fig. 1), indicating that beneath the columns, the surface level exceeds that of the non-irradiated surface.

The cross-section SEM micrographs of the imprints

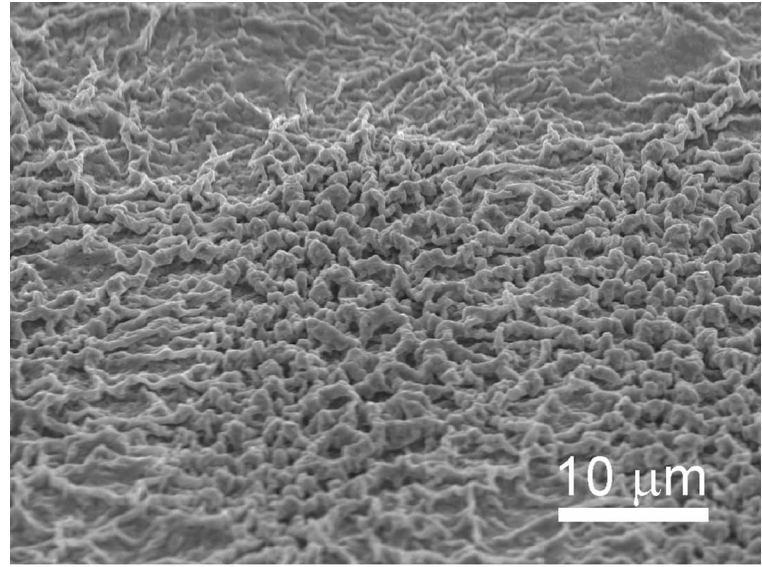

(a)

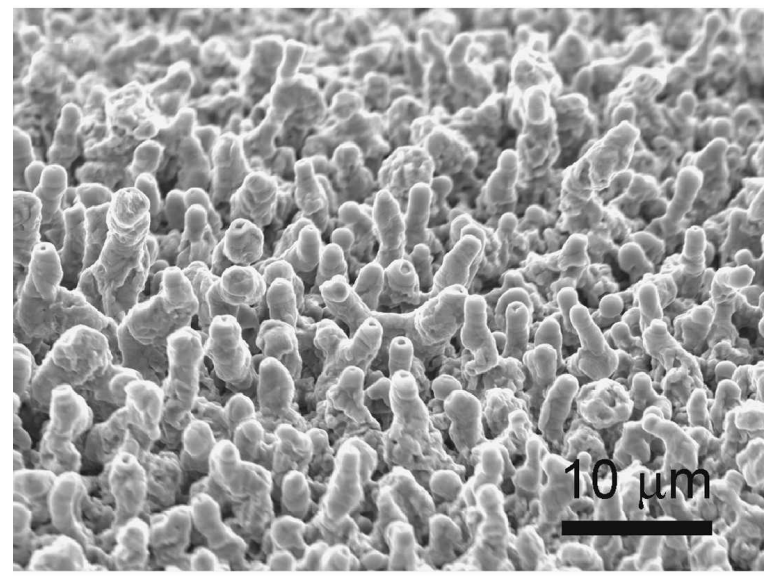

(b)

FIG. 1. Surface SEM micrographs of samples obtained under unbiased conditions with (a) 810 and (b) 1500 laser pulses. The samples were tilted $50^{\circ}$.

obtained with 1200 and 1500 laser pulses show the above described transition from an almost plane surface to a central depression (Fig. 3). Moreover, the affected area beneath the microcolumns arrays is porous, reaching a depth of about $20 \mu \mathrm{m}$.

\section{B. Biased titanium targets}

The SEM micrographs corresponding to the central part of the imprints obtained with 810 laser pulses and many bias voltages are presented in Fig. 4. On the surface of the reference imprint (unbiased target), it can be observed that besides the initial structuring, smooth areas remain with a morphology still similar to that of the non-irradiated target. Nevertheless, under biased conditions (both negative and positive) the surface structuring process becomes accelerated. In addition, under negative bias the rate of the columns growth is higher than under positive bias [compare Figs. 4(c) and 4(d)]. Indeed, under negative $500 \mathrm{~V}$ target bias, the surface becomes completely covered by a dense columnar structure similar to that shown in Fig. 1(b) for unbiased target with much 


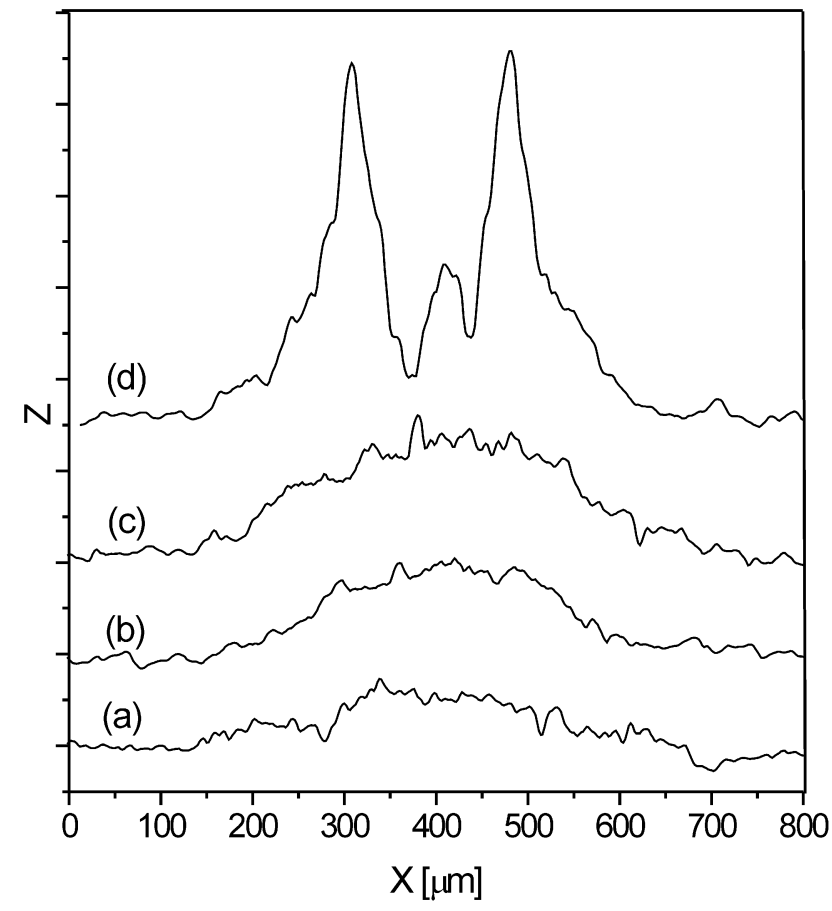

FIG. 2. Surface profiles of samples obtained under unbiased conditions with (a) 810 , (b) 900 , (c) 1200 , and (d) 1500 pulses. The distance between the ticks on the ordinate axis corresponds to $10 \mu \mathrm{m}$.

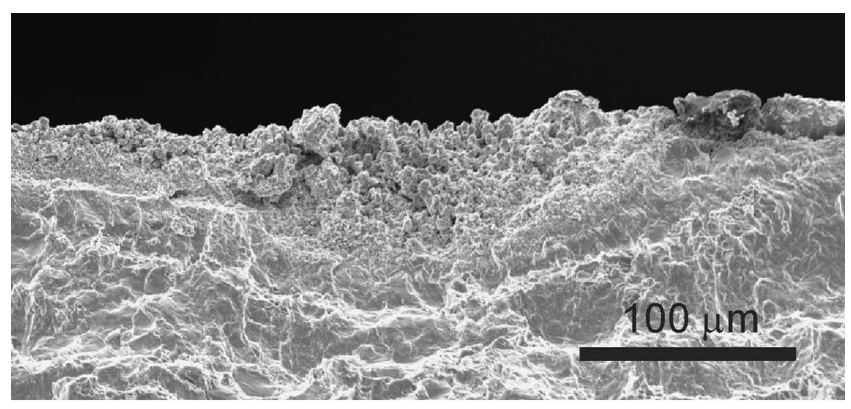

(a)

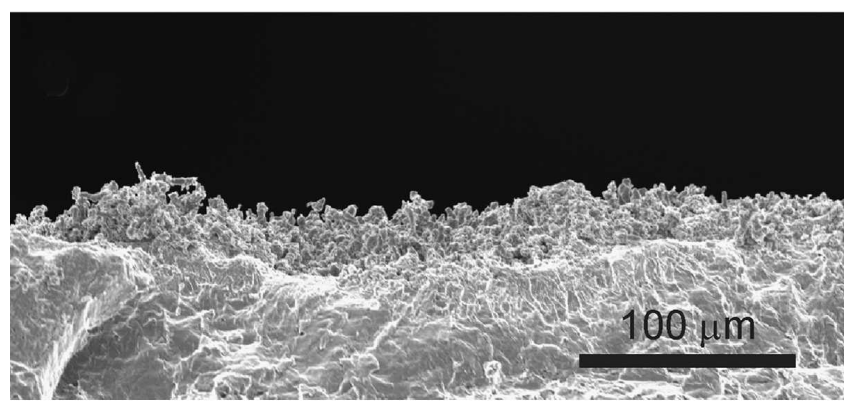

(b)

FIG. 3. Cross-section SEM micrographs of samples obtained under unbiased conditions with (a) 1200 and (b) 1500 pulses.

more pulses, while under the same but positive bias value, smooth surface areas can still be observed. We remark that even if in the SEM images in normal view it cannot be observed, the morphology of the microcolumns grown on biased targets is similar to that of those grown in unbiased conditions [Fig. 1(b)].

The profiles across the diagonal of the full laser imprints obtained with 810 and 1200 pulses and many bias voltages are presented in Fig. 5. Applying a positive or negative target bias voltage of $500 \mathrm{~V}$ during the irradiation with 810 laser pulses increases the overall elevation of the laser-imprinted area. At 1200 laser pulses, the increase of the elevation could be observed even at lower, 100 and $250 \mathrm{~V}$, target bias voltage. Moreover, at negative $100 \mathrm{~V}$ bias voltage, the increase of the elevation is more pronounced at the borders, while a crater formation takes place in the center of the imprints. At higher target bias voltage values, the crater formation can also be observed at positive bias. However, the craters are always deeper in the negative as compared to the positive-bias case [compare Fig. 5(g) with 5(h) and Fig. 5(i) with $5(j)]$. This last feature can also be observed in the cross-section SEM images of the imprints obtained with 1200 laser pulses grown on positively and negatively biased targets (Fig. 6). We mention that similar crater formation can be observed at higher number of pulses also under unbiased conditions. Moreover, in this case the crater bottoms always remain higher than the level of the non-irradiated target surface.

\section{DISCUSSION}

The origin of the rippled wavelike structures [Fig. 1(a)], which precede the column growth is associated with the onset of significant target nitridation, i.e., with hydrodynamic instabilities driven by surface tension forces with positive temperature gradients of the surface tension coefficient induced by the local nitrogen concentration gradient on the target surface. ${ }^{2,12}$ Since the rippled structure formation takes place in the absence of the laser generated plasma, it must not be influenced by the external electric field produced by the target bias.

The evolution of the target morphology with the increase of the number of laser pulses from this initial rippled wavelike structure to a dense columnar microrelief [Fig. 1(b)] is attributed to non-uniform target vaporization and selective vapor recondensation on the molten columns top. ${ }^{2,13}$ Indeed, multiple reflections of the laser light on the ripples walls lead to preferential vaporization from the valleys. ${ }^{14}$ In addition, the incident energy density is higher in the valleys perpendicular to the laser beam than on the vertical walls, contributing to the nonuniform target vaporization. ${ }^{15}$ The nonuniform surface vaporization in turn could induce local pressure gradients in the laser generated plasma developing in front of the irradiated target, the pressure being higher in the valleys than on the ripples top. ${ }^{16}$ These pressure gradients could induce liquid motion from the valleys toward the columns top. 


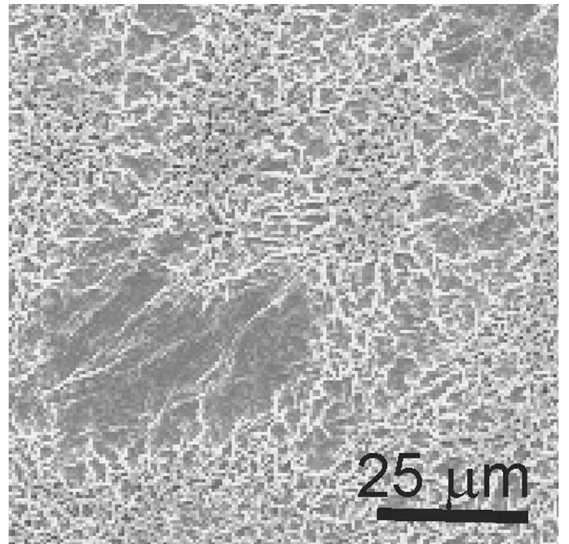

(a)

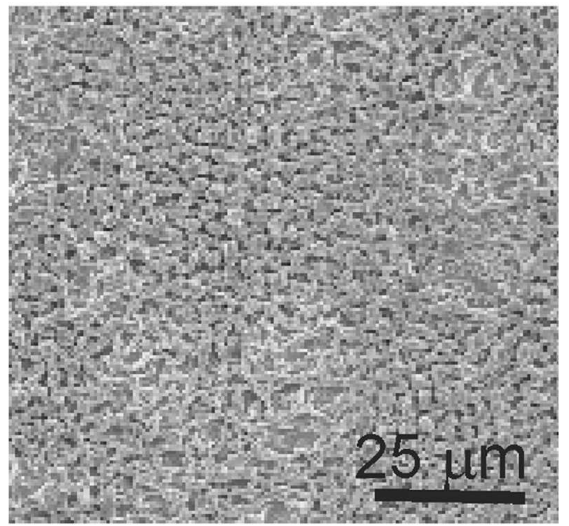

(c)

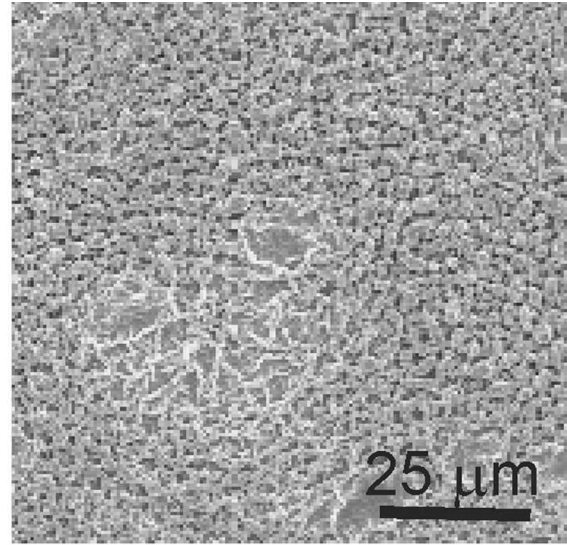

(b)

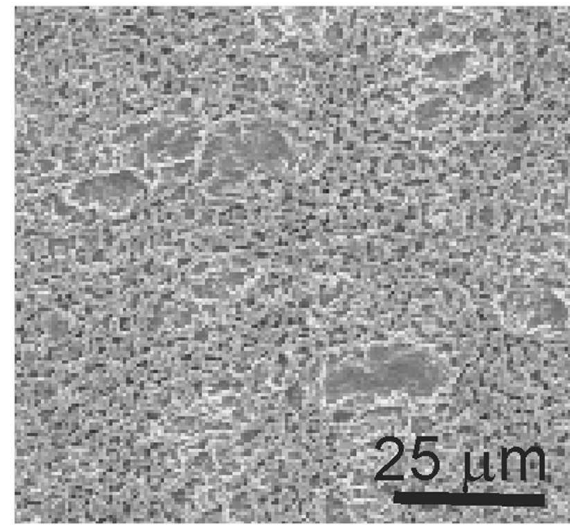

(d)

FIG. 4. Surface SEM micrographs of samples obtained with 810 laser pulses and a target bias voltage of (a) $0 \mathrm{~V}$, (b) $-250 \mathrm{~V}$, (c) $-500 \mathrm{~V}$, and (d) $+500 \mathrm{~V}$. The samples were not tilted.

The application of a target bias voltage generates an electric field, which exerts an extra driving force upon the species present in the laser produced ablation plasma. Indeed, under negative target bias, the positively charged particles (the ions present in the ablation plasma) are attracted toward the surface. These positive ions come from ejected particles from the target and nitrogen molecules ionised by collisions. ${ }^{1}$ The negatively charged particles, mainly electrons, are repelled by the target. Their acceleration increases their ionization capability, resulting in an enhancement of the plasma ionisation, and thus of the ion bombardment in the target. In the case of positive bias voltage, electrons are pulled back to the target, and positive ions are accelerated toward the direction opposite the target. This increases their ionization capability and also leads to the enhancement of the plasma ionization. In addition, under both positive and negative target bias, the neutral species, which are dipoles induced by the electric field, are also attracted towards the target, but with a lower intensity. All these processes take place during but also after the end of the laser pulses since high ambient pressure limits the expansion and enhances the duration of the laser generated plasma. $^{1}$
The impact energy of the accelerated plasma species under both positive and negative target bias is transferred to the target surface producing an increase of its temperature. This increase favors the reactive gas incorporation and surface compound formation, i.e., nitridation reaction. ${ }^{17,18}$ Thus, at a given number of pulses, both positive and negative target bias could be considered equivalent, in a sense, to the unbiased case with additional pulses. Indeed, these additional pulses would have similar effects, i.e., increased target temperature and nitrogen incorporation, ${ }^{2,3}$ leading to enhanced structure formation. Finally, due to the significant increase of the target temperature, the melting-vaporization without preferential redeposition sites leads to crater formation in the center of the irradiated area (Figs. 5 and 6).

Moreover, the applied electric field under negative target bias conditions can directly contribute to the growth of the columns by vapor recondensation. Indeed, the electric field is more intense on the tops of the columns, ${ }^{19}$ which represent preferential redeposition sites for the ionic component of the laser generated plasma. On the other hand, however, at higher bias voltages, the ions accelerated in the laser plasma could sputter the target surface. ${ }^{20-22}$ This effect could already be present at 


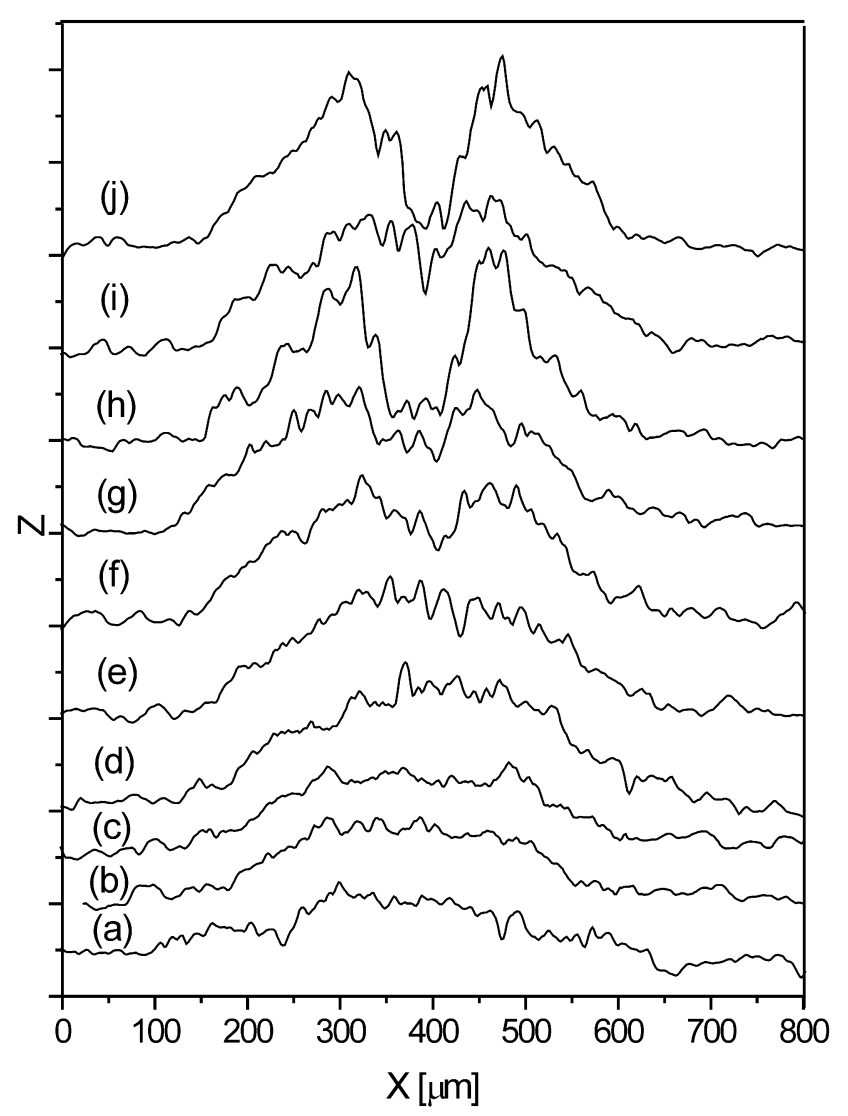

FIG. 5. Surface profiles of samples obtained with 810 laser pulses and a target bias voltage of (a) $0 \mathrm{~V}$, (b) $+500 \mathrm{~V}$, and (c) $-500 \mathrm{~V}$; and with 1200 laser pulses and a target bias voltage of (d) $0 \mathrm{~V}$, (e) $+100 \mathrm{~V}$, (f) $-100 \mathrm{~V},(\mathrm{~g})+250 \mathrm{~V}$, (h) $-250 \mathrm{~V}$, (i) $+500 \mathrm{~V}$, and (j) $-500 \mathrm{~V}$. The distance between the ticks on the ordinate axis corresponds to $10 \mu \mathrm{m}$.

$500 \mathrm{~V}$ negative target bias and would act against the columns growth.

Our results showed that both positive and negative target bias voltage lead to an enhanced surface structuring and column growth as compared to the unbiased condition (Figs. 4 and 5). Considering all the described mechanisms, this can be attributed to the increase of the reactivity of the species that form the titanium nitride microcolumns, due to the increase of the plasma ionization and to the higher target temperature produced by the impact of the accelerated plasma species.

In addition, the column growth was faster under negative target bias than it was under positive target bias. This could be attributed to the preferential vapor recondensation of the ionic component of the laser plasma on the top of the initial columns, where the electric field has the maximum value. However, it should also be considered that the described processes (increase of target temperature and plasma ionization degree, and thus enhanced nitridation reaction on the target surface) can have different intensities for the same positive or negative bias voltage value. Then it is not possible to unambiguously

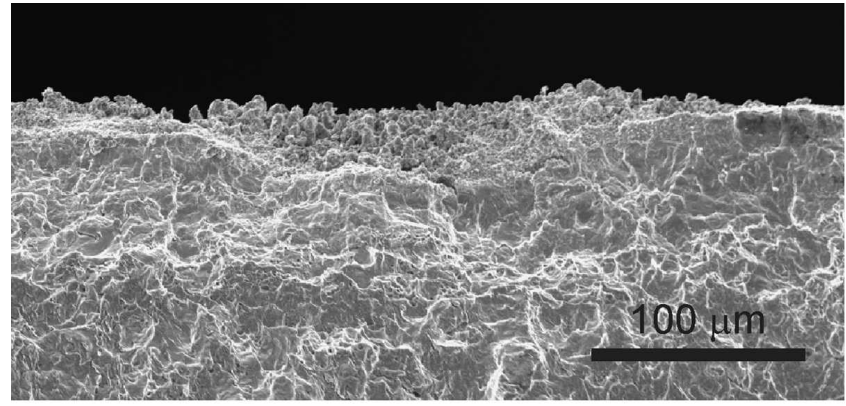

(a)

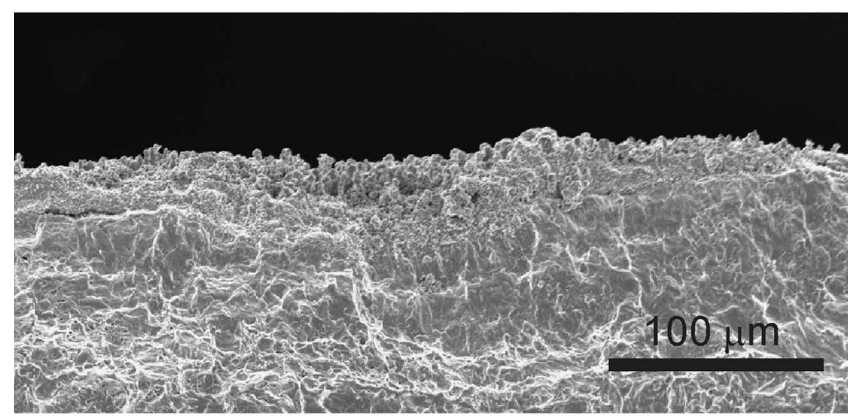

(b)

FIG. 6. Cross-section SEM micrographs of samples obtained with 1200 laser pulses and a target bias voltage of (a) $+250 \mathrm{~V}$ and (b) $-250 \mathrm{~V}$.

attribute the cause of the observed difference between the behaviours with positive and negative target bias to the first effect.

\section{CONCLUSION}

Titanium nitride microcolumns were grown under the action of cumulative Nd:YAG $(\lambda=1.064 \mu \mathrm{m}, \tau \sim 300$ $\mathrm{ns}, v=30 \mathrm{kHz}$ ) laser irradiation of titanium targets in high-pressure nitrogen. Comparative studies of surface structuring were performed for unbiased and biased targets. We found that the applied bias voltage enhances the development of the specific columnar morphology. The specific structure formation was associated with laser induced surface compound formation and hydrodynamic instabilities, as well as selective evaporation and redeposition of plasma species. Our results showed that the surface structuring process is more accelerated under negative than it is under positive target bias. The preferential vapor condensation of the ionic component of the plasma on the top of columns when negatively biased could contribute to this difference. However, due to the complexity of the processes that lead to the column growth, it is difficult to clearly delimit the mechanisms and distinguish which one plays the decisive role. 


\section{ACKNOWLEDGMENTS}

This work is part of a program supported by Ministerio de Ciencia y Tecnología (MCYT) of the Spanish Government (MAT2001-3757), Fondo Europeo de Desarrollo Regional (FEDER), and Departament d'Universitats, Recerca i Societat de la Informació (DURSI) of the Catalan Government (2001SGR00080).

\section{REFERENCES}

1. D. Bauerle: In Laser Processing and Chemistry (Springer-Verlag, Berlin, Germany, 2000).

2. E. György, A. Pérez del Pino, P. Serra, and J.L. Morenza: Microcolumns development on titanium by multi-pulse laser irradiation in nitrogen. J. Mater. Res. 18, 2228 (2003).

3. E. György, A. Pérez del Pino, P. Serra, and J.L. Morenza: Influence of the ambient gas in laser structuring of the titanium surface. Surf. Coat. Technol. 187, 245 (2004).

4. S.I. Dolgaev, S.V. Lavrishev, A.A. Lyalin, A.V. Simakin, V.V. Votonov, and G.A. Shafeev: Formation of conical microstructures upon laser evaporation of solids. Appl. Phys. A: Mater. Sci. Proc. 73, 177 (2001).

5. Y. Kawakami and E. Ozawa: Tungsten microcone arrays grown using nanosecond pulsed Nd:YAG laser in a low-pressure He-gas atmosphere. Appl. Phys. A: Mater. Sci. Proc. 74, 59 (2002).

6. F. Sanchez, J.L. Morenza, and V. Trtik: Characterization of the progressive growth of columns by excimer laser irradiation of silicon. Appl. Phys. Lett. 75, 3303 (1999).

7. A.J. Pedraza, J.D. Fowlkes, and D.H. Lowndes: Silicon microcolumn arrays grown by nanosecond pulsed-excimer laser irradiation. Appl. Phys. Lett. 74, 2322 (1999).

8. V. Oliveira and R. Vilar: Characterization of columns grown during $\mathrm{KrF}$ laser micromachining of $\mathrm{Al}_{2} \mathrm{O}_{3}-\mathrm{TiC}$ ceramics. J. Mater. Res. 18, 1123 (2003).

9. A. Wennerberg, A. Hallgren, C. Johansson, and S. Danelli: A histomorphometric evaluation of screw-shaped implants each prepared with two surface roughnesses. Clin. Oral Impl. Res. 9, 11 $\underline{(1998)}$.
10. M. Nakamoto and K. Fukuda: Field electron emission from $\mathrm{LaB}_{6}$ and TiN emitter arrays fabricated by transfer mold technique. Appl. Surf. Sci. 202, 289 (2002).

11. E. György, I.N. Mihailescu, P. Serra, A. Pérez del Pino, and J.L. Morenza: Single-pulse Nd:YAG laser irradiation of titanium: Influence of laser intensity on surface morphology. Surf. Coat. Technol. 154, 63 (2002).

12. A.I. Nwobu, R.D. Rawlings, and D.R.F. West: Nitride formation in titanium based substrates during laser surface melting in nitrogen-argon atmospheres. Acta Mater. 47, 631 (1999).

13. P.R. Willmott and J.R. Huber: Pulsed laser vaporization and deposition. Rev. Mod. Phys. 72, 315 (2000).

14. L.K. Ang, Y.T. Lau, R.M. Gilgenbach, and H.L. Spindler: Analysis of laser absorption on a rough metal surface. Appl. Phys. Lett. 70,696 (1997).

15. W.W. Duley: UV Lasers: Effects and Applications in Materials Science (Cambridge University Press, Cambridge, U.K., 1996)

16. A.B. Brailovsky, S.V. Gaponov, and V.I. Luchin: Mechanisms of melt droplets and solid-particles ejection from a target surface by pulsed laser action. Appl. Phys. A: Mater. Sci. Proc. 61, 81 (1995).

17. M. Wautelet and L.D. Laude: Cohesion of solids under laser irradiation. Appl. Phys. Lett. 36, 197 (1980).

18. J.M.E. Harper, J.J. Cuomo, and H.T.G. Hentzell: Synthesis of coumpund thin films by dual ion beam deposition. I. Experimental approach. J. Appl. Phys. 58, 550 (1985).

19. B.W. Han, H.S. Lee, and B.T. Ahn: Field-emission characteristics of $\mathrm{CoSi}_{2} / \mathrm{TiN}$-coated silicon emitter tips. Appl. Surf. Sci. 187, 45 (2002).

20. A.D. Akhsakhalyan, Yu.A. Bityurin, S.V. Gaponov, A.A. Gudkov, and V.I. Luchin: Processes occurring in an erosion plasma during laser vacuum deposition of films. I. Properties of a laser erosion plasma in the inertial-expansion stage. Sov. Phys. Tech. Phys. 27, 969 (1982).

21. S.V. Gaponov, B.M. Luskin, B.A. Nesterov, and N.N. Salashchenko: Morphological features and structures of films condensed from a laser plasma. Sov. Phys. Solid State 19, 1736 (1977).

22. R. Kelly and A. Miotello: Mechanisms of pulsed laser sputtering, in Pulsed Laser Deposition of Thin Films, edited by D.B. Chrisey and G.K. Hubler (John Wiley, New York, NY, 1994) pp. 55-88. 